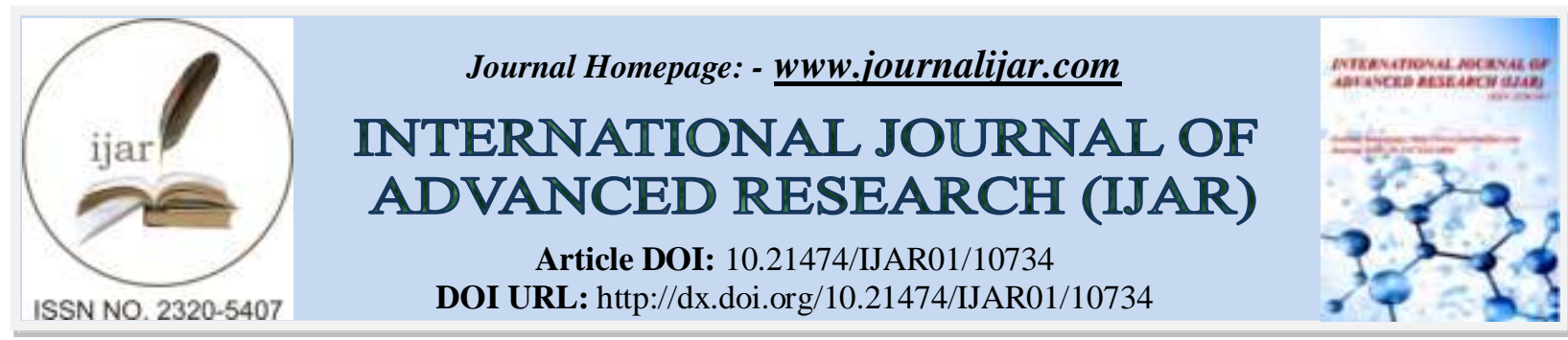

RESEARCH ARTICLE

\title{
SEMINAL VESICLE CYST WITH IPSILATERAL RENAL AGENESIS (ZINNER SYNDROME): CASE REPORT
}

Jihane Habi, Sophia Berrada, Hind Guerroum, Mariam Kassimi, Nabil Chikhaoui and Mohamed Mahi

Department of Radiology, Cheikh Khalifa Hospital / Mohammed VI University of Health Sciences, Casablanca, Morocco.

\section{Manuscript Info}

………………....

Manuscript History

Received: 25 January 2020

Final Accepted: 27 February 2020

Published: March 2020

Key words:-

Seminal Vesical Cyst, Diagnostis, Imaging

\section{Abstract}

The seminal vesicle cyst associated with agenesis of the kidney and homolateral ureter is a rare defect known as Zinner syndrome. We report a case of a 41-years-old patient with symptoms of lower abdominal pain associated with hemospermia. Within our radiology department, he underwent an ultrasound exam, CT scan and then an MRI which revealed Zinner's syndrome on the left side. The objective of our work is to analyze the interest of each means of imaging in the diagnosis of Zinner syndrome.

Copy Right, IJAR, 2020,. All rights reserved.

\section{Introduction:-}

Zinner's syndrome is defined by a seminal vesicle cyst associated with ipsilateral renal agenesis. Its frequency is very rare; the reported frequency is approximately $0.0046 \%$ [1]. It is found in young patients during a period of sexual activity. Its clinical symptomatology is varied, based mainly on urogenital symptoms. The different means of imaging play a very important role in its positive diagnosis.

\section{Case Report:-}

41-years-old patient, with no particular pathological history, he presented with lower abdominal pain associated with hemospermia.

A diagnostic pelvic ultrasound was performed first, which showed the presence of a left retro-vesical oval cystic formation, anechoic with thin-walled. For better visibility of the abdomen, a CT scan was performed which revealed left renal agenesis and a right kidney with normal size and morphology, in addition to retro-vesical cystic formation, hyperdense, multi-lobulated, without contrast enhancement and thin wall (Figure 1).

A pelvic MRI of characterization of the retro vesical cystic formation has been released and showed a formation of the left seminal vesicle, multi lobulated, measured at $37 \times 33 \mathrm{~mm}$, with intermediate signal in $\mathrm{T} 2$ and hypersignal in T1, without taking any particular contrast. This cystic formation continues with a terminal oblong structure, testifying to a seminal vesicle cyst with an ectopic termination of the left ureter (Figure 2).

Corresponding Author:- Jihane Habi

Address:-Departement of Radiology, Cheikh Khalifa Hospital/ Mohammed VI University of Health Sciences, Casablanca, Morocco. 

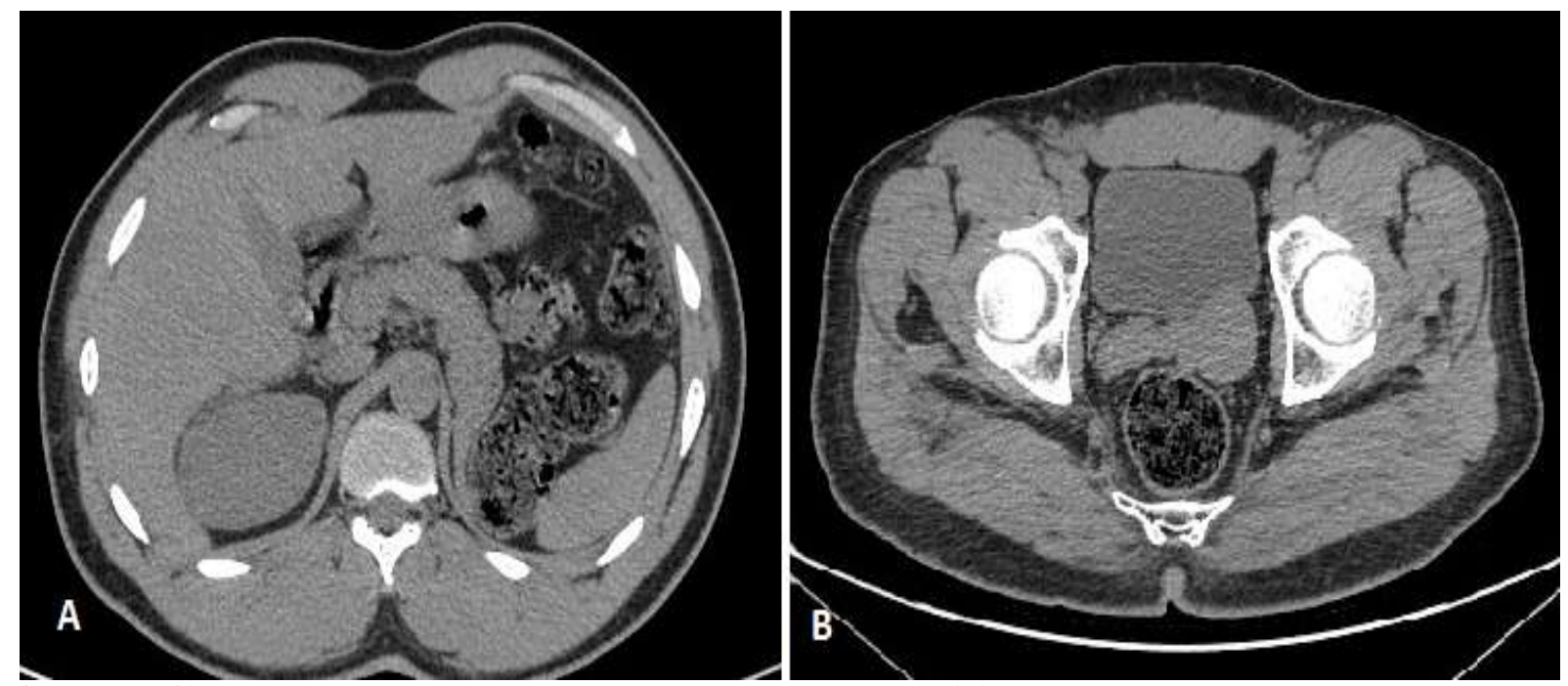

Figure 1:- (A): CT axial section showing agenesis of the left kidney. (B): Axial CT scan without injection showing the hyperdense seminal vesicle cyst compared to the density of urine.
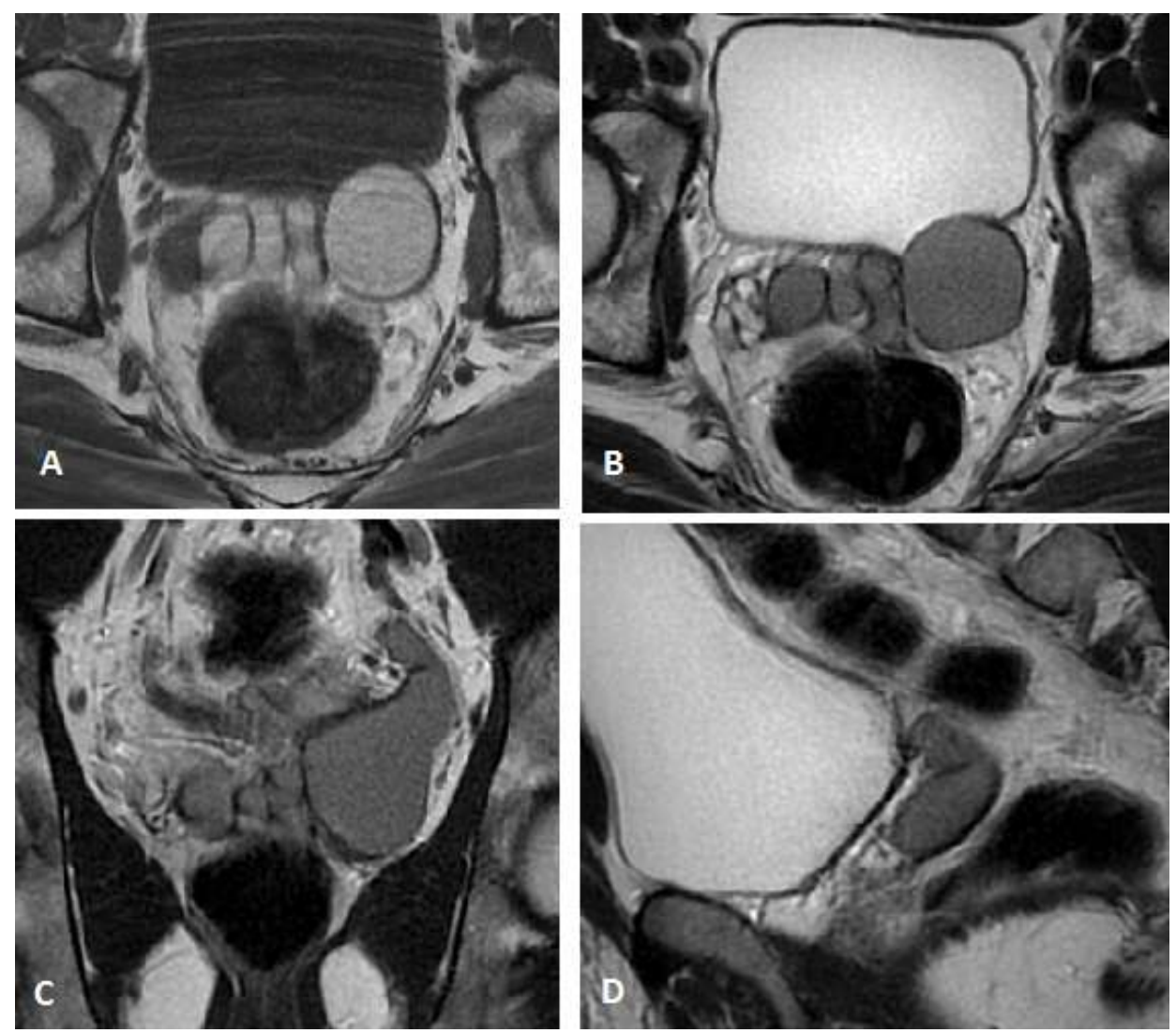

Figure 2:- Seminal vesicle cyst in T1 (A) and T2 (B). The ureter ectopic termination in coronal T2 section (C) and sagittal T2 section (D).

\section{Discussion:-}

Zinner's syndrome is an uncommon birth defect, described like a seminal vesicle cyst associated with ipsilateral renal agenesis and obstruction of the ejaculatory duct. 
The association between this triad of malformation is due to their common embryological origin (Mesonephric or Wolffian duct).

Maldevelopment of the distal mesonephric duct results in absence of the ureteral bud, and, therefore, ipsilateral renal agenesis or dysplasia in addition to atresia of the ejaculatory duct with subsequent obstruction of the seminal vesicles and formation of cysts. [2]

The syndrome was first described by Zinner since 1914 [3]. Since then, it has often been reported on the right and rarely on the left.

This syndrome is the prerogative of patients between the 2 nd and the 5 th decade, at the beginning of sexual activity by distention of the seminal vesicle or during an assessment of infertility. Its discovery is often fortuitous on clinical examination with digital rectal examination by the palpation of a mass in retro prostatic region or during a radiological exam when the cyst of the seminal vesicle is of size $<5 \mathrm{~cm}$. If the size of the cyst is $>5 \mathrm{~cm}$, it can be discovered by multiple and varied urogenital symptoms (Pain pelvic, dysuria, pain on ejaculation, hemospermia, infertility or even abdominal pain).

Cysts of the seminal vesicles greater than $12 \mathrm{~cm}$ have been termed "giant" cysts and often are present with symptoms of bladder and colonic obstruction because of mass effect [4].

Imaging is more than enough to make a positive diagnosis.

Ultrasound provides information on the nature of the cyst, its retro-vesical location, its size and appearance (An anechoic pelvic mass with a thick, irregular wall and occasional wall calcifications, or the mass may contain internal debris reflecting prior hemorrhage or infection [5]. On sonography, ipsilateral kidney is absent, and the obstructed ejaculatory ducts are seen as anechoic structures in the pelvis, though hemorrhage or infection can produce internal low level echoes) [6]. For our patient, pelvic ultrasound could only detect the retro-vesical mass.

Vasovesiculography is an invasive means of imaging which consists of injecting the iodized contrast and checking under scope the passage of the product towards the ectopic ureteral bud testifying to an obstruction of the ejaculatory duct. This examination was not released for our patient due to its very invasive nature.

CT is much more reliable than ultrasound to show ipsilateral renal agenesis. Reported findings have been variable ranging from a cystic pelvic mass with a thick irregular wall to a solid mass and apparent enlargement of the ipsilateral seminal vesicle [7]. The solitary cysts had irregular margins $(9 / 14,64.3 \%)$ or tubulosaccular shape $(5 / 14$, $35.7 \%$ ) [8].The cyst density was slightly greater than that of urine with an average CT value of $8 \pm 5$ HU (range: $3-$ $15 \mathrm{HU}$ ) and no contrast enhancement [8]. It can also have a spontaneously hyperdense content if its content is highly protein like the aspect that we found in our patient.

MRI is the modality of choice for precise delineation of the anatomy of the male genital tract, for demonstrating the seminal vesicles and evaluating anomalies of the mesonephric duct and to differentiate the seminal vesicle cysts from other cystic pelvic masses [9]. The seminal vesicle cyst is generally hyposignal in T1 and hypersignal in T2 without contrast enhancement. Old hemorrhage and a high proteinaceous concentration in the seminal fluid with old spermatozoa are established features of seminal vesicle cysts; these conditions shorten T1 time and account for the high signal intensity [10]. It also shows the termination of the ectopic ureter into the seminal vesicle and the dilation of the ejaculatory duct.

\section{For the treatement:}

Asymptomatic patients have conservative treatment. This conservative approach will help to prevent possible side effects (e.g. erectile dysfunction) from surgical intervention. [11]

For symptomatic patient, invasive treatments including transurethral resection of the ejaculatory duct (TURED), exploration, laparoscopic and robotic vesiculectomy are suggested [12]. Clogging the ejaculatory duct can solve the problem of infertility. 


\section{Conclusion:-}

Zinner's syndrome is a rare birth defect that often affects the right side. We have presented on this article a case of this syndrome with left involvement which is even rarer. Imaging alone can make a positive diagnosis. MRI is the examination of choice because of its ability to establish a positive diagnosis and pre-surgical anatomical mapping.

\section{References:-}

1. Azhar Farooqui and al (2018); Massive seminal vesicle cyst with ipsilateral renal agenesis - Zinner syndrome in a Saudi patient, Departments of Internal Medicine and 1 Urology, Suleiman Al Habib Medical Group, Riyadh, Saudi Arabia Urology Annals

2. Livingston L, Larsen CR (2000). Seminal vesicle cyst with ipsilateral renal agenesis. AJR Am J Roentgenol; 175:177-80.

3. Mehdi KP, Nasser S. (2013) Management of Zinner's syndrome associated with contralateral seminal vesicle hypoplasia: a case report. Case Rep Urol.:1e3.

4. Heaney JA, Pfister RC, Meares EM Jr (1987); Giant cyst of the seminal vesicle with renal agenesis. AJR;149:139-140

5. King BF, Hattery RR, Lieber MM, et al. (1991) ; Congenital cystic disease of the seminal vesicle. Radiology; 178:207-11.

6. Trigaux JP, Van Beers B, Delchambre F. (1991); Male genital tract malformations associated with ipsilateral renal agenesis: sonographic findings. J Clin Ultrasound; 19:3e10.

7. Kenney PJ, Leeson MD (1983); Congenital anomalies of the seminal vesicles: spectrum of computed tomographic findings. Radiology; 149:247-251

8. Xiao-Song Jiang1 and al, (2018); Zinner's syndrome: clinical features and imaging diagnosis . Asian Journal of Andrology 20, 316-317

9. Arora SS, and al (2007); CT and MRI of congenital anomalies of seminal vesicles. AJR Am J Roentgenol;189:130e5

10. Huan-Wu Chen, Sheng-Chau Huang and al (2006) ; Magnetic Resonance Imaging of Seminal Vesicle Cyst Associated with Ipsilateral Urinary Anomalies. Elsevier

11. Gorrea M, Lorente R, Roel J. (2001) ; Seminal vesicle cyst associated with ipsilateral renal agenesis and papillary carcinoma of the bladder. Eur Radiol; 11:2500-3.

12. Pereira BJ, Sousa L, Azinhais P, et al. (2009); Zinner's syndrome: an up-todate review of the literature based on a clinical case. Andrologia; 41:322-330. 